STUDI

FRANCESI
Studi Francesi

Rivista quadrimestrale fondata da Franco Simone

190 (LXIV | I) | 2020

Varia - fasc. I - gennaio-aprile 2020

\title{
Balzac et l'étranger, sous la direction de F. SPANDRI
}

\section{Marion Mas}

\section{CpenEdition \\ Journals}

\section{Édition électronique}

URL : https://journals.openedition.org/studifrancesi/22871

DOI : 10.4000/studifrancesi.22871

ISSN : 2421-5856

\section{Éditeur}

Rosenberg \& Sellier

\section{Édition imprimée}

Date de publication : 1 avril 2020

Pagination : 193-194

ISSN : 0039-2944

\section{Référence électronique}

Marion Mas, «Balzac et l'étranger, sous la direction de F. SPANDRI », Studi Francesi [En ligne], 190 (LXIV I I) | 2020, mis en ligne le 01 mai 2020, consulté le 03 août 2021. URL : http://journals.openedition.org/ studifrancesi/22871; DOI : https://doi.org/10.4000/studifrancesi.22871

Ce document a été généré automatiquement le 3 août 2021.

\section{(c) (†)}

Studi Francesi è distribuita con Licenza Creative Commons Attribuzione - Non commerciale - Non opere derivate 4.0 Internazionale. 


\title{
Balzac et l'étranger, sous la direction de F. SPANDRI
}

\author{
Marion Mas
}

\section{RÉFÉRENCE}

Balzac et l'étranger, sous la direction de F. SPANDRI, “The Balzac review / Revue Balzac" 1, 2018, Paris, Classiques Garnier, 210 pp.

1 Quoi d'étonnant que le premier numéro de la "The Balzac review / Revue Balzac", au «profil résolument international», fût consacré au thème de «l'étranger»? Cette livraison, dirigée par Francesco Spandri, aborde dans sa variété une notion importante, qui n'a été que ponctuellement traitée par les études balzaciennes. Le volume fait jouer les relations entre Balzac et l'étranger suivant deux axes. D'une part, il examine la fécondité du regard porté depuis l'étranger sur l'œuvre d'un écrivain qui avait luimême l'ambition d'une renommée internationale. D'autre part, il interroge les représentations de l'étranger dans l'œuvre romanesque et journalistique, en mettant en évidence leurs enjeux littéraires et idéologiques.

2 L'étranger, commence par rappeler Francesco SPANDRI (Introduction. Balzac, la France et l'étranger, pp. 13-24), comporte à la fois une dimension géographique, une dimension sociale et une dimension fantasmatique. Ces aspects organisent la vision que l'œuvre balzacienne porte sur les espaces européen et extra-européen. Plus fondamentalement, Francesco Spandri montre que l'intérêt de Balzac pour l'étranger découle d'un partipris épistémologique issu des Lumières, où la relativité constitue une dimension essentielle des sociétés et des faits humains: «c'est cette variation dans l'espace des opinions et des conduites qui captive Balzac» (p. 16). L'étranger constitue un élément déterminant de la réflexion et de l'écriture balzaciennes, car il offre un regard décalé sur la France contemporaine. C'est cet aspect, bien plus que la dimension pittoresque, qui intéresse Balzac. Les contributions de Misha AVREKH et de Roland LE HUENEN montrent ainsi combien les relations de voyage dans la correspondance ou la Lettre sur 
Kiev (1847) sont des anti-récits de voyage, se désintéressant des descriptions des villes et des monuments, et mettant l'accent essentiellement sur les aspects pratiques (lenteur des transports, tracasseries administratives etc.). Si ces traits sont évidemment liés à la logique affective qui gouverne ces textes (ce qui importe à l'épistolier est de rejoindre «l'Étrangère» au plus vite), ils soulignent aussi, en creux, que le regard du voyageur se porte avant tout sur des aspects sociologiques et politiques, occasionnant des comparaisons avec la France. L'évocation de l'étranger, avec Balzac, apparaît d'abord comme un mouvement de décentrement.

3 Ainsi, la contribution de Thomas PAVEL Historicism and Beyond (pp. 25-36), montre que l'essai d'Ernst Curtius, Balzac (1923), a permis de rompre avec une tradition critique française centrée sur une approche documentaire de l'œuvre, perpétuée jusqu'à Brunetière au moins, pour mettre en évidence un Balzac spirituel, romantique et mystique. Curtius a été l'un des premiers à mettre au jour le rôle essentiel de l'énergie chez les personnages balzaciens, et à analyser dans le détail les thèmes universels constituant la dynamique profonde de l'œuvre: l'énergie, la passion, le pouvoir et la connaissance. L'article d'Andrew waTTS, Balzac British Style. Avarice, Austerity and the Tight Spaces of Rex Tucker's "Eugénie Grandet" (BBC2, 1965-1966) (pp. 37-54) montre que l'adaptation télévisée d'Eugénie Grandet, par ses choix de mise en scène, d'une part, met en lumière certains aspects du roman, et d'autre part, offre un éclairage renouvelé sur le contexte politique et social de l'Angleterre des années 1960. Le réalisateur Rex Tucker a su exploiter les contraintes techniques de la télévision (caméras lourdes et difficilement déplaçables, usage du gros plan) pour souligner la valeur symbolique du confinement de l'espace et celle de l'escalier, renvoyant à la fois à l'avarice de Grandet, à son ascension sociale et à La Divine Comédie de Dante. En retour, cette adaptation actualise la fiction balzacienne, en mettant en garde contre les dangers liés à un libéralisme économique effréné, au sortir de l'austérité d'après-guerre.

Les deux articles suivants, Balzac, voyages et impressions de Russie, de Pologne et d'Ukraine (1843, 1847-1850) (pp. 55-70) et Balzac's Forecasts. Familiarity, Predictability and Numbers (pp. 71-86), s'attachent à l'appréhension paradoxale de l'étranger dans la correspondance et dans la Lettre sur Kiev. Roland LE HUENEN montre qu'en marge de notations relevant d'«une topographie sentimentale et toute personnelle» (p.56), se développe un portrait politique de la Russie évoluant au fil des voyages: tandis que le séjour de 1843 développe une critique voilée de l'autoritarisme tsariste, la période de 1847 à 1850 marque au contraire une fascination appuyée pour le pouvoir fort. La singularité de ces récits de voyage engage également une réflexion sur un genre galvaudé. Mettant en évidence la difficulté à sortir des lieux communs, les lettres balzaciennes opèrent une critique plus profonde, fondée «sur la double opposition privé/public et réel/fictif» (p.63) et sur la marge de manœuvre critique du locuteur: «prendre pour objet le réel de l'historien, en l'assumant à titre privé, conduit à s'exposer» (p. 64). En s'appuyant sur l'abondance des chiffres et des comptes qui saturent la correspondance, Misha AVREKH montre que la relation à l'étranger se traduit par une écriture de la statistique, l'étranger désignant alors aussi bien l'ailleurs géographique que la province du Curé de village ou du Médecin de campagne. Les chiffres, langage universel, permettent d'apprivoiser l'altérité. Ainsi, dans les débuts de leur relation épistolaire, le rapport détaillé de ses dépenses et de ses recettes que fait Balzac à Mme Hanska sert à créer l'illusion d'une intimité partagée. Plus généralement, les choses comptables (les prix, les salaires, les distances, les durées de voyage...) offrent 
une prise à la compréhension de l'étranger car elles peuvent être rapportées à du connu. En revanche, lorsque Balzac se trouve placé face à de l'incommensurable, l'étranger demeure incompréhensible. Les chiffres sont donc aussi un moyen de faire face à l'instabilité et à l'imprévisibilité, en donnant un sentiment de maîtrise.

Les contributions de la dernière partie du dossier examinent dans quelle mesure la représentation de l'étranger offre un point de vue oblique sur la France de la Monarchie de Juillet. Dans If fossils could talk (pp. 87-98), Dorian BeLL propose de lire la matière égyptienne d'Une passion dans le désert comme la figuration du poids, sur le présent, d'une série de brouillages épistémologiques (entre humanité et animalité, entre masculin et féminin, entre sauvage et civilisé, et entre plusieurs strates temporelles - la temporalité mythique de la conquête napoléonienne, la temporalité archaïque de l'histoire naturelle de Cuvier, le passé récent de la conquête coloniale algérienne et l'actualité parisienne de l'argent, de l'ambition et de la finance). Les effets de détour et de réversibilité mis en scène par le texte de Balzac établissent alors un lien entre la figure du Juif et les aventures financières de la colonisation, qui devient, au cours du siècle, un motif antisémite important. Takao TASHIWAGI, dans La Chine dans "L'Interdiction" de Balzac (pp.99-120), examine les sources érudites de la nouvelle, et montre que la philosophie chinoise informe la construction du personnage du marquis d'Espard, et à travers lui, un modèle aristocratique idéal recélant une dimension politique. Dans La France et l'étranger. Les articles de politique extérieure (pp. 121-134), Aude DÉRUELLE examine un corpus peu étudié par la critique balzacienne. Qualifiant d'«envers gris de l'œuvre romanesque» (p.123) les articles de politique extérieure des Lettres sur Paris publiées dans "Le Voleur" entre 1830 et 1831, de La Chronique de Paris (1836) et des Lettres russes parues dans la "Revue parisienne" (1840), elle montre que cet ensemble met en relief l'évolution politique de Balzac gommée par l'œuvre romanesque, d'un libéralisme condamnant le congrès de Vienne à un légitimisme recherchant un nouvel équilibre européen, où la France aurait une place prépondérante (p.132) et révèle, par rapport à celle-ci, «une autre face de la médiocrité de la France bourgeoise» (p. 128). Ainsi, l'analyse balzacienne estime que la politique extérieure de la Monarchie de Juillet, caractérisée par une paralysie de l'action, révèle une vision étriquée de la France. Enfin, tout en rappelant les dispositifs énonciatifs particuliers de ces trois séries d'articles, Aude Déruelle montre que s'y façonne progressivement une écriture journalistique singulière, «au creuset de l'idéologie» (p. 123).

6 À la lecture de ce dossier, force est de constater l'importance du thème politique, qui traverse la quasi-totalité des contributions, fût-ce au titre de remarques périphériques. De fait, c'est, écrit Francesco Spandri, «en se faisant terrain et discours politique que l'étranger devient explicitement un objet de connaissances» (p. 19). Une autre ligne de force du dossier consiste en la réflexion sur les stratégies déployées pour écrire sur l'étranger, que mettent en évidence les articles. Faute de place, nous ne pouvons que mentionner les trois varia et l'«archive» qui clôturent la revue: Philippe BERTHIER, dans L'étranger absolu (pp.139-150), montre que Louis Lambert décline le motif de la séparation comme manifestation de la pensée et de ses effets. Mariolina BERTINI, dans Désirs, indices, secrets. La lanterne magique de la princesse de Cadignan analyse Les Secrets de la princesse de Cadignan (pp. 151-168) comme l'une des sources profondes de La Recherche du temps perdu. Dans Balzac et l'honorable député (pp. 169-178), Peter вRоoкs interroge les rapports entre roman et journal à partir d'une apparente contradiction entre la trame d'Illusions perdues et la préface à la troisième partie du roman. Enfin Susi PIETRI présente 
un essai de William Butler Yeats intitulé Louis Lambert, originellement publié dans le “London Mercury” en juillet 1934 (pp. 193-204). 